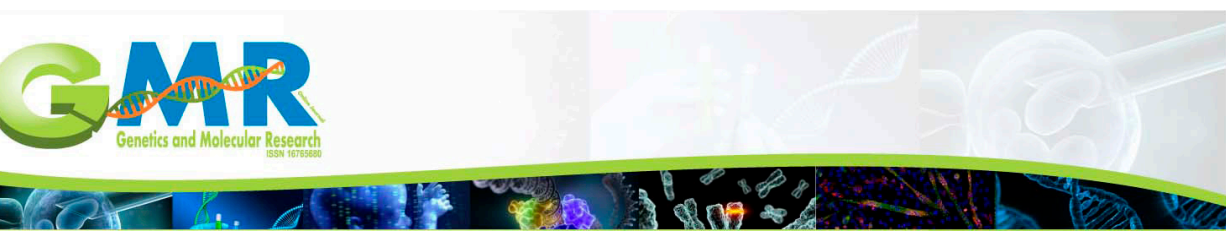

\title{
Serum cytokine modulation after Staphylococcus hyicus infection in $\mathrm{BALB} / \mathrm{c}$ mice
}

Y. Li ${ }^{1}$, L. Wang ${ }^{1,2}$, L.Y. Zhang ${ }^{1}$, Y.L. Liu' ${ }^{1}$, Z.Y. Jiang ${ }^{1}$, R.J. Cai ${ }^{1}$ and C.X. Song ${ }^{2}$

${ }^{1}$ Institute of Animal Health, Guangdong Academy of Agriculture Sciences,

Guangzhou, China

${ }^{2}$ Guangdong Open Laboratory of Veterinary Public Health,

Guangdong Provincial Key Laboratory of Livestock Disease Prevention, Institute of Animal Health, Guandong Academy of Agricultural Sciences,

Guangzhou, China

Corresponding author: C.X. Song

Email: songchangxcn@163.com

Genet. Mol. Res. 14 (4): 16682-16693 (2015)

Received August 27, 2015

Accepted October 24, 2015

Published December 11, 2015

DOI http://dx.doi.org/10.4238/2015.December.11.16

ABSTRACT. Staphylococcal infection is a severe bacterial infection. Finding satisfactory predictable biological markers is essential for the treatment of this condition. In this study, we applied a 32-marker sandwich ELISAbased antibody array to evaluate cytokine changes in Staphylococcus hyicus-inoculated BALB/c mice at 24 and $48 \mathrm{~h}$ post infection. Among the cytokines detected, the expression levels of granulocyte colony stimulating 
factor (G-CSF), interleukin 6 (IL-6), macrophage inflammatory protein 2, and keratinocyte chemoattractant $(\mathrm{KC})$ were increased to levels more than twice higher than those in the control group $24 \mathrm{~h}$ after infection, while the expression of interleukin 12p40p70 (IL-12p40p70) was decreased to less than half the level measured in the controls. The expression of G-CSF, IL-6, monocyte chemoattractant protei-5, chemokine ligand 11, and $\mathrm{KC}$ was upregulated $48 \mathrm{~h}$ post infection, whereas IL-12p40p70 expression was still significantly lower $(P<0.05)$. Among the detected cytokines, the expression levels of G-CSF, IL-6, and KC were constantly upregulated, while IL-12p40p70 was downregulated. This result was then validated by an ELISA assay analysis, which confirmed that G-CSF, IL-6, KC, and IL12 p40p70 expression levels were specifically modulated after an S. hyicus bacterial infection, while granulocyte monocyte colony stimulation factor, IL-12, and IFNy levels were significantly increased after a viral infection. Our study indicated the potential of cytokines G-CSF, IL-6, KC, and IL12 p40p70 as markers for detecting $S$. hyicus infection. The results of this study may provide useful data for the appropriate use of medication following $S$. hyicus infection.

Key words: Staphylococcus hyicus; Cytokine; Antibody array; Mice; Disease marker

\section{INTRODUCTION}

Staphylococcus hyicus is a coagulase-variable species comprising both coagulase-positive and coagulase-negative isolates. S. hyicus, producing exfoliative toxins (ETs), is primarily known as the main causative agent of exudative epidermitis (EE) in piglets (Tanabe et al., 1996; Andresen, 2005). The disease is characterized by the separation of the cells in the upper stratum of the epidermis, exfoliation of the skin, erythema, and the formation of a thick, greasy, brown exudation (L'Ecuyer and Jericho, 1996; Ahrens and Andresen, 2004). S. hyicus has been isolated from pigs with septic polyarthritis and bovine mastitis and from White Leghorn laying chickens experiencing skin and feather problems (Phillips et al., 1980; Roberson et al., 1996; Chenier and Lallier, 2012). To the best of our knowledge, two cases of human infection have been reported: as early as 1997 , the first case of a wound infection following a donkey bite was reported (Osterlund and Nordlund, 1997); recently, S. hyicus was isolated from a septic patient-a farmer who had been in close contact with piglets (Casanova et al., 2011). ETs produced by staphylococci are reported to be involved in some subcutaneous infections in mammals. In S. hyicus, five different ETs, including SHETB, ExhA, ExhB, ExhC, and ExhD, have been reported, with amino acid sequences homologous to ETA and ETB of Staphylococcus aureus, as they all belong to the serine protease-like group of ETs (Ahrens and Andresen, 2004; Futagawa-Saito et al., 2007; Nishifuji et al., 2008; lyori et al., 2010). It was reported that the four Exh isoforms selectively digest a single peptide bond in the extracellular region of swine desmoglein 1 (Dsg1), a desmosomal cadherin-type cell-cell adhesion molecule, inducing skin exfoliation in piglets (Fudaba et al., 2005; Tosi, 2005).

Cytokines are a heterogeneous group of soluble small polypeptide or glycoprotein mediators, forming part of a complex network that helps regulate the immune and inflammatory 
responses (Hill and Martins, 2006). Many biological processes such as apoptosis, inflammation, angiogenesis, immune response, and migration often accompany changes in cytokine expression levels. Immune response also varies between pathogenic insults, such as bacterial infection or viral infection. Cytokines mediate the activity of Th1 cells that induce cell immunity from viral infections or of Th2 cells that induce humoral immunity in defense against bacterial infections (Zhao et al., 2012).

Because of the extensive cross-communication between cytokines, multiplex assays must be used to obtain a complete picture of the biological responses and functions. Cytokines are known to play many critical roles in innate and adaptive immunity, apoptosis, angiogenesis, cell growth, and differentiation (Tosi, 2005; Jin et al., 2008; de la Fuente et al., 2009; McDonald et al., 2011). Detection of expression levels of multiple cytokines can provide new insights into the development of $S$. hyicus infection and help discover key factors in pathogenic progress.

Antibody array technology has drawn great attention due to its high-throughput, flexibility, and cost-effectiveness. The three platforms for antibody array approaches include sandwich-based, label-based, and competition-based antibody arrays (Borrebaeck and Wingren, 2007). Antibody array technology has emerged as a promising technology for disease biomarker discovery. Moreover, antibody array screening improves the chances of discovering key factors, disease mechanisms, and biomarkers related to cytokine signaling (Kim et al., 2009; Jiang et al., 2013). Cytokine levels have been assessed in S. aureus- infected mice to find biomarkers that indicate potentially fatal outcomes following infection (van den Berg et al., 2013). However, until now, little was known about cytokine expression profiles during S. hyicus infections.

In this study, sera from BALB/c mice infected with S. hyicus were screened for 32 different cytokines at prescribed time points during infection. Protein expression assays were used to determine changes in the cytokine profile after infection.

\section{MATERIAL AND METHODS}

\section{Bacteria}

The clinical strain S. hyicus ZC-4 (JQ728535) was used. This strain was isolated in pure culture from the pericardial fluid of a diseased piglet with serious EE from a conventional pig-rearing farm in the Guangdong province of China. Notably, S. hyicus ZC-4 was a virulent strain carrying the exfoliative toxin ExhA. Cultures of S. hyicus grown in nutrient broth were stored at $-80^{\circ} \mathrm{C}$.

\section{Animals and experimental design}

Specified opportunistic pathogen-free female BALB/c S. hyicus-free mice (four weeks old on the day of infection), purchased from Southern Medical University, were used. All procedures were in accordance with the guidelines for the care and use of laboratory animals by the Institute of Experimental Animals of Guangdong Academy of Agriculture Sciences. Experiments were performed in two groups. In the control group of 16 mice, each mouse received a $0.2 \mathrm{~mL}$ subcutaneous injection of phosphate buffered saline (PBS). In the $S$. hyicus treated group of 16 mice, each mouse received $S$. hyicus at a dose of $1.0 \times 10^{8} \mathrm{CFU}$ via subcutaneous injection. All mice were closely monitored throughout the study for clinical signs. At both 24 and $48 \mathrm{~h}$ after 
infection, eight mice were sacrificed via decapitation for organ extraction, bacterial culture, and serum collection. The serum samples were stored at $-80^{\circ} \mathrm{C}$ until needed. The livers, spleens, kidneys, and lungs were removed from the infected mice at different time points after infection, and organ homogenates were examined for bacterial presence.

\section{Identification of the clinical isolates}

DNAwasisolatedusing the Easy-DNA ${ }^{\mathrm{TM}}$ gDNAPurificationKit(Invitrogen, Carlsbad, CA, USA) following the manufacturer protocols. Specific primers (5'-ATGGTTTTGGTAGAATTGGTCGTTTA-3') and (5'-GACATTTCGTTATCATACCAAGCTG-3') were used to amplify a 931 bp gap gene. The DNA was amplified by polymerase chain reaction (PCR), with an initial denaturation at $94^{\circ} \mathrm{C}$ for 5 min, followed by 30 cycles at $94^{\circ} \mathrm{C}$ for $30 \mathrm{~s}, 56^{\circ} \mathrm{C}$ for $1 \mathrm{~min}, 72^{\circ} \mathrm{C}$ for $30 \mathrm{~s}$, and finally, extension at $72^{\circ} \mathrm{C}$ for $10 \mathrm{~min}$.

\section{Examination of the genes encoding staphylococcus exfoliative toxins by PCR}

The PCR was performed according to the general steps using the primers 5'-ATAGAGGAGAAATCAACATG-3' (forward) and R 5'-CTATAGTTACTTGACCTCTA-3' (reverse) to amplify an $865 \mathrm{bp}$ exfoliative toxin ExhA gene. The cycling conditions included an initial denaturation at $94^{\circ} \mathrm{C}$ for $4 \mathrm{~min}$, followed by 35 cycles at $94^{\circ} \mathrm{C}$ for $30 \mathrm{~s}, 56^{\circ} \mathrm{C}$ for $30 \mathrm{~s}, 72^{\circ} \mathrm{C}$ for 1 min, and finally, extension at $72^{\circ} \mathrm{C}$ for $10 \mathrm{~min}$.

\section{Cytokine antibody array analysis}

The following cytokines were assessed in the sera of mice infected with S. hyicus at 24 and 48 h after infection: granulocyte colony stimulating factor (G-CSF), granulocyte monocyte colony stimulating factor (GM-CSF), interferon (IFN) $\gamma$, tumor necrosis factor (TNF) $\alpha$, tissue inhibitor of metalloproteinase 1 (TIMP-1), Leptin (Ob), thrombopoietin (TPO), thymus activation regulation chemokines (CCL17), stem cell factor (SCF; Kit ligand), soluble TNF receptor type 1 (sTNF R1; TNFRS1A), and the interleukins (IL) -2, IL-3, IL-4, IL-5, IL-6, IL-9, IL-10, IL-12p40, IL12p70, IL-13, and IL-17. The following chemokines were selected: keratinocyte chemoattractant (KC; CXCL1), monocyte chemoattractant protein 1 (MCP-1; CCL2), MCP-5 (CCL12), macrophage inflammatory protein 1a (MIP-1a; CCL3), MIP-2 (CXCL2), MIP-3 $\beta$ (CCL19), the chemokine regulated-upon-activation normal T-cell expressed and secreted (RANTES; CCL5), Eotaxin (CCL11), cutaneous T-cell-attracting chemokine (CTACK/CCL27), secondary lymphoid tissue chemokine (SLC; 6Ckine; CCL21), and vascular endothelial growth factor (VEGF). Selected cytokines and chemokines were representative of both pro- and anti-inflammatory cytokines. Cytokine levels were determined as per the protocol for using the RayBiotech mouse cytokine antibody array G2 (AAM-CYT-G2-4; RayBiotech, Norcross, GA, USA). The array consisted of glass slides that were coated with antibodies, including the positive and negative controls. The signals were scanned with a GenePix 4000B fluorescence laser scanner (Axon Instruments, Foster City, Cal.). The results were analyzed with the RayBiotech analysis tool specifically designed to analyze RayBiotech G-Series Antibody Arrays (RayBiotech Inc, Norcross, GA, USA). The signals were normalized using the positive and negative controls included on the array. 


\section{Enzyme-linked immunosorbent assays (ELISA)}

ELISA was performed according to the RayBio® ELISA manual (RayBiotech, Inc., Norcross, GA, USA).

\section{Statistical analysis}

The data are expressed as the mean \pm SEM. The data were analyzed using the unpaired two-tailed Student $t$-test for comparison between two groups. Differences with a value of $\mathrm{P}<0.05$ were considered statistically significant.

\section{RESULTS}

\section{Clinical signs}

All challenged mice displayed a range of clinical signs $24 \mathrm{~h}$ after infection, including depression, mild inflammatory exudation, rough fur coats and fur loss. Forty-eight hours after infection, all of the inoculated mice exhibited typical clinical signs, obvious inflammatory exudation, and serious hair loss. All control mice remained healthy during the study period.

\section{Isolation and identification of S. hyicus}

A strain of Staphylococcus was isolated in pure culture from the mouse organs and identified as $S$. hyicus by morphological and biochemical examination. The isolate displayed the morphology of Staphylococcus under the microscope, were shaped as gram-positive cocci in clumps, and showed the biochemical characteristics of $S$. hyicus, such as glucose decomposition and a positive outcome in the indole test. To confirm the results of the biochemical tests, we analyzed the S. hyicus ZC-4 special gene at a molecular level using PCR, examined the gap gene in the control, and the exfoliative toxins in the bacterium.

\section{Cytokine arrays results}

The serum levels of the 32 selected cytokines were assessed using antibody array technology. The reproducibility for the glass-slide-based arrays was assessed by testing replicate aliquots of the samples with two sub-arrays printed on the same slide and assayed at the same time. After subtracting background signals, the duplicate values were averaged and normalized with reference to positive controls. The comparison of signal intensities for antigen-specific antibody spots in array images of each cytokine between mice infected with $S$. hyicus and the control group was used to determine relative differences in expression levels. In this study, all cytokines with a significant difference $(P$ $<0.05)$ in expression were selected. Then, any $\geq 2$-fold increase or $\geq 2$-fold decrease $(\leq 0.5$ of the expression level of control mice) in signal intensity for a single cytokine between mice infected with $S$. hyicus and the control group was considered a measurable and significant difference in expression. Significant differences are observed between S. hyicus-infected mice versus mice in the control group $24 \mathrm{~h}$ and $48 \mathrm{~h}$ after $\mathrm{S}$. hyicus infection; the data are shown in Table 1. Data are expressed as 2-, 4-, 6-, 8-fold or greater increases, or 2-fold decreases, in mice infected with S. hyicus over control mice. 
Twenty-four hours after inoculation, the levels of 4 out of 32 cytokines (G-CSF, IL-6, KC [CXCL1], MIP-2 [CXCL1]) were at least twice higher compared to those of the controls. The G-CSF level in serum was 193.81 times higher than that of the controls. The level of IL-6 increased 6.2-fold, KC 24.74-fold, and MIP-2 (CXCL1) 3.8-fold compared to those of the controls. Among the cytokines detected, only the expression of IL-12p40p70 was significantly decreased. Expression of IL-12p40p70 in the infected mice was only 0.41 times the level of the control $24 \mathrm{~h}$ after infection (Table 1 and Figure 1).

Table 1. Changes in the level of serum cytokines in BALB/c mice in response to $S$. hyicus infection $(P<0.05)$.

\begin{tabular}{|c|c|c|}
\hline \multirow[t]{2}{*}{ Cytokine } & \multicolumn{2}{|c|}{ Fold change (ZC-4/PBS) } \\
\hline & 24 hours post infection & 24 hours post infection \\
\hline 6Ckine & 0.88 & 1.08 \\
\hline СТACK & 0.85 & 1.16 \\
\hline CCL11 & 1.25 & $2.69 \uparrow$ \\
\hline G-CSF & $193.81 \uparrow \uparrow \uparrow \uparrow$ & $343.41 \uparrow \uparrow \uparrow \uparrow$ \\
\hline GM-CSF & 0.85 & 0.94 \\
\hline IL-2 & 0.94 & 1.14 \\
\hline IL-3 & 0.81 & 0.99 \\
\hline IL-4 & 0.97 & 0.91 \\
\hline IL-5 & 0.86 & 0.96 \\
\hline IL-6 & $6.20 \uparrow \uparrow \uparrow$ & $2.44 \uparrow$ \\
\hline IL-9 & 0.69 & 1.07 \\
\hline IL-10 & 0.88 & 0.97 \\
\hline IL-12p40 p70 & $0.41 \downarrow$ & $0.47 \downarrow$ \\
\hline IL-12p70 & 0.77 & 1.03 \\
\hline IL-13 & 0.81 & 1.09 \\
\hline IL-17 & 0.96 & 1.01 \\
\hline IFN-y & 0.87 & 0.87 \\
\hline $\mathrm{KC}$ & $24.74 \uparrow \uparrow \uparrow \uparrow$ & $6.30 \uparrow \uparrow \uparrow$ \\
\hline Leptin (Ob) & 0.69 & 1.08 \\
\hline MCP-1/CCL2 & 0.66 & 1.27 \\
\hline MCP-5/CCL12 & 1.45 & $3.43 \uparrow$ \\
\hline MIP-1 alpha/CCL3 & 1.12 & 1.21 \\
\hline MIP-2/CXCL1 & $3.80 \uparrow$ & 1.68 \\
\hline MIP-3 beta/CCL19 & 0.93 & 0.98 \\
\hline RANTES/CCL5 & 0.84 & 0.98 \\
\hline SCF/Kit Ligand (KITLG) & 1.34 & 0.98 \\
\hline sTNFR1 & 1.05 & 1.19 \\
\hline sTNFR2 & 1.15 & 0.94 \\
\hline TIMP-1 & 1.52 & 1.90 \\
\hline TNF- $\alpha$ & 0.99 & 0.96 \\
\hline Thrombopoietin & 1.01 & 1.07 \\
\hline VEGF & 0.50 & 1.22 \\
\hline
\end{tabular}

Expression of cytokines in the serum at two time points after infection. The controls are BALB/c mice injected with PBS. Values are expressed as $2,4,6$, or $\geq 8$ times greater or lower than controls: $\uparrow 2$ times greater than control; $\uparrow \uparrow 4$ times greater than control; $\uparrow \uparrow \uparrow 6$ times greater than control; $\uparrow \uparrow \uparrow \uparrow 8$ times greater than control; $\downarrow 0.5$ times lower than control.

Forty-eight hours after infection, the expression levels of 5 of 32 cytokines (G-CSF, IL-6, KC [CXCL1], CCL11, and MCP-5 [CCL12]) were significantly higher than the control levels. The amount of G-CSF was 343.41 -fold higher than in the control. The level of IL- 6 was 2.44 -fold that of the control. KC expression was 6.30 -fold higher than that of the control. CCL11 levels were elevated 2.69-fold compared to those in the controls. MCP-5 (CCL12) levels were increased 3.43fold over those in the control. Expression of IL-12p40p70 was only 0.47 times the level of the control at $48 \mathrm{~h}$ after infection (Figure 2 and Table 1).

From the data above, G-CSF, IL-6, KC (CXCL1), and IL-12p40p70 were consistently at significantly different levels in comparison to the controls at both 24 and $48 \mathrm{~h}$ after infection. 

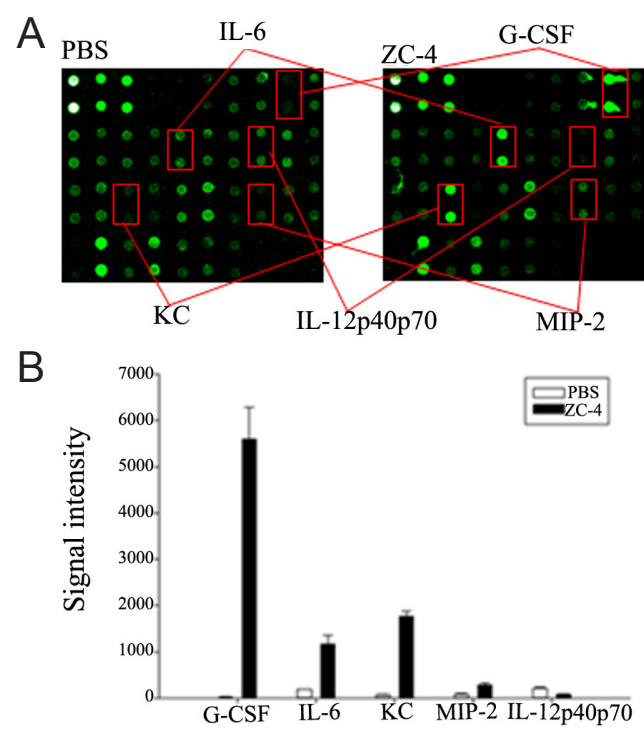

Figure 1. Cytokine profiles in sera from BALB/c mice with $S$. hyicus and control mice at 24 hours after infection. A. Representative cytokine antibody arrays. The spots for G-CSF, IL-6, KC, MIP-2 and IL-12p40p70 showed a marked density increase (or reduction) compared to the control. B. Expression of cytokines was found to be 2 -fold higher or lower than that of the controls. All $P<0.05$.

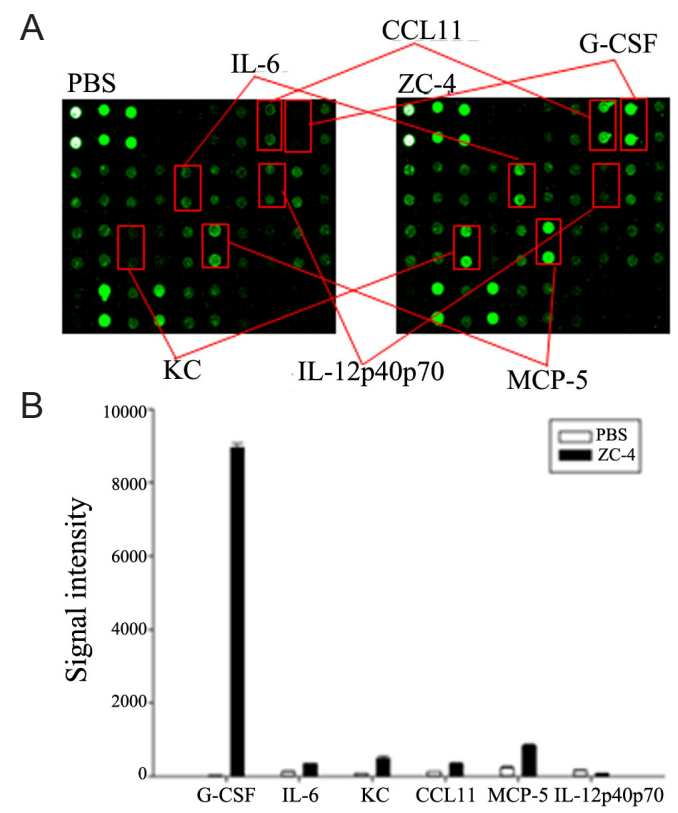

Figure 2. Cytokine profiles in sera from BALB/c mice with $S$. hyicus and control mice at $48 \mathrm{~h}$ after infection. A. Representative cytokine antibody arrays. The spots for G-CSF, IL-6, KC, CCL-11, MCP-5, and IL-12p40p70 showed a marked density increase (or reduction) compared to the control. B. Expression of cytokines was found to be at least twice higher or less than that of the controls. All $\mathrm{P}<0.05$. 


\section{Validation of cytokines with the ELISA assay}

To confirm our observations from the cytokine antibody array studies, an ELISA assay was used to quantitatively measure the expression levels of G-CSF, IL-6, KC, and IL-12p40p70. At both 24 and $48 \mathrm{~h}$ after infection, the IL-6, KC, and G-CSF expression levels were all significantly upregulated in the sera of mice inoculated with $S$. hyicus compared to the controls, as indicated in Figure 3. For IL-12p40p70 detection, the expression level was also significantly downregulated. Figure 3 shows representative ELISA data for cytokines IL-6, KC, G-CSF, and IL-12p40p70 24 h after infection.

IL-6

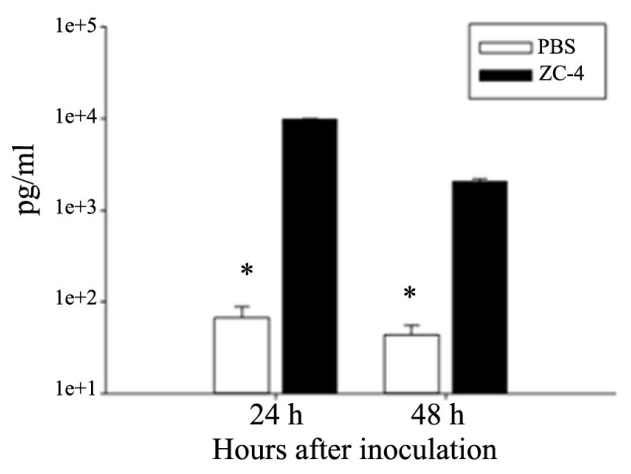

G-CSF

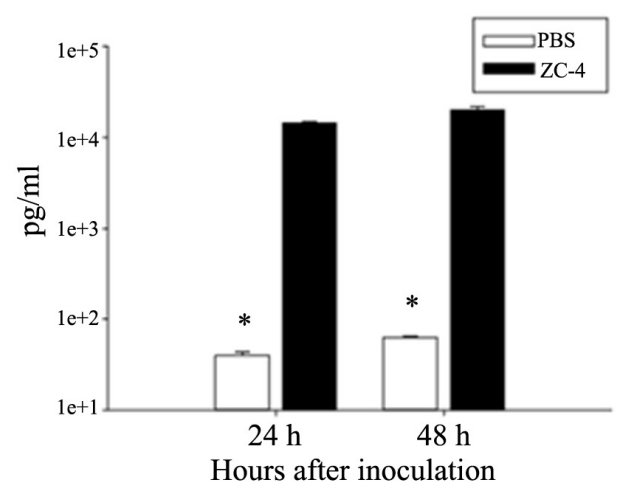

$\mathrm{KC}$

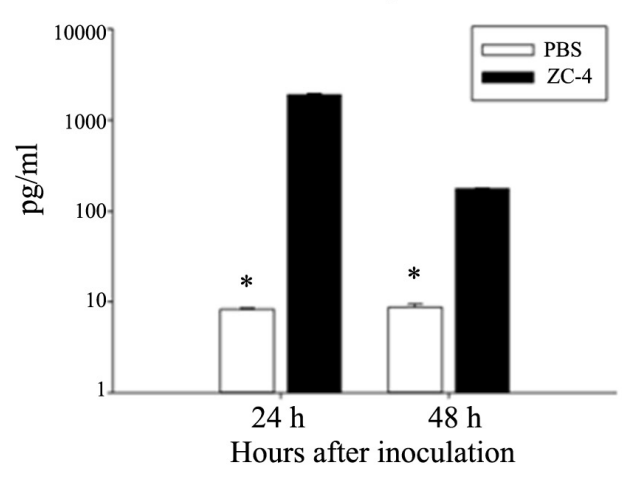

IL-12p40p70

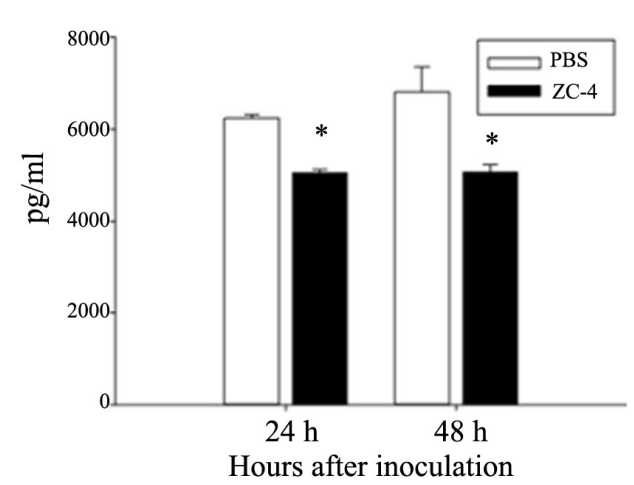

Figure 3. IL-6, KC, G-CSF, and IL-12p40p70 expression in sera of S. hyicus-infected mice compared to controls. For IL-6, KC, G-CSF, and IL-12p40p70, animals at all times post-infection had significantly different $\left({ }^{*}\right)$ cytokine levels compared to controls. ELISA data are shown as the mean protein concentration $(\mathrm{pg} / \mathrm{mL})$. ${ }^{*} \mathrm{P}<0.05$.

\section{DISCUSSION}

Staphylococcus hyicus is the main causative agent of EE in piglets (Tanabe et al., 1996; Andresen, 2005). In this study, an S. hyicus-infected model was established in BALB/c mice. The 
cytokine expression profile in sera from $S$. hyicus infected in BALB/c mice was then analyzed by the semi-quantitative, sandwich-based antibody array technology.

Our study is the first report of the application of sandwich-based antibody array technology for cytokine detection in the sera of BALB/c mice inoculated with $S$. hyicus. Among the 32 cytokines detected, levels of CCL11, G-CSF, IL-6, KC, MIP-2/CXCL1, and MCP-5/CCL12 increased to at least twice those of the controls and levels of IL-12p40p70 decreased to less than half that of the controls at either 24 hours or 48 hours post infection. Among the above cytokines, G-CSF, IL-6, and $\mathrm{KC}$ were consistently upregulated at the two time points studied, and IL-12p40p70 was consistently downregulated. These results were validated with an ELISA assay.

Interleukin 6 (IL-6) is a pleiotropic cytokine involved in the regulation of acute-phase reaction, inflammation, hematopoiesis, and the transition from innate to acquired immunity (Fielding et al., 2008; Abajo et al., 2012). Endogenous IL-6 plays a crucial anti-inflammatory role in both local and systemic acute inflammatory responses in mouse models. The viable bacterial count in the livers of IL-6-deficient mice was higher than those of control mice (Onogawa et al., 2013). Furthermore, elevated IL-6 levels in the serum and increased expression of IL-6 mRNA in the skin were detected in response to $S$. aureus ETA exposure (Dalrymple et al., 1996). G-CSF is a pleiotropic cytokine best known for its specific effects on the proliferation, differentiation, and activation of hematopoietic cells of the neutrophilic granulocyte lineage (Cheers et al., 1988; Rolle et al., 2013). In vitro, G-CSF stimulates growth, differentiation, and other functions in cells of the neutrophil lineage (Kawakami et al., 1990; Demetri and Griffin, 1991). KC, a member of the alpha (CXC) chemokine sub-family, is the murine functional homologue of human IL-8 (Kawakami et al., 2004), and appears to have an important role in inflammation, based on the pattern of KC expression in a number of inflammatory disease models. Similar to our findings, KC was assessed in a study of $S$. aureus infection in mice and reported as one of the biomarkers predicting fatal outcome of infection (van den Berg et al., 2013).

IL-12 was the only cytokine to decrease to less than half the level of the control at each time point. IL-12 is produced by macrophages and B-lymphocytes and has been shown to have multiple effects on T cells and natural killer (NK) cells. These effects include inducing production of IFN-y and TNF by resting and activated T and NK cells, synergizing with other IFN- $y$ inducers at both the transcriptional and post-transcriptional levels (Bozic et al., 1995; Une et al., 2003). It is likely that the amplification of antigen-specific responses by IL-12 may be at least partially impaired by decreased IL-12, thus contributing to decreased IFN-Y production, although the difference was not significant.

Recently, the up-regulation of several factors including TNF- $\alpha$, IL-1 $\alpha$, G-CSF, IL-6, MIP-1a, and KC after S. aureus infection was reported (van den Berg et al., 2013). Our study identifies four cytokines (G-CSF, IL-6, KC, and IL-12p40p70) that may represent useful markers for detecting $S$. hyicus infection. Cytokine modulation is crucial in activating immune responses to various pathogens. Further studies are required to arrive at a conclusion and to detect biomarkers that predict the severity of infection and its progression.

Cytokines play a critical role in the host's immune defense against infection. It has been established that intracellular pathogens, such as those involved in a viral infection, initiate a cellular immune response characterized by the activation of type $1 \mathrm{~T}$ lymphocytes (T1) and the production of IFN-y, TNF- $\alpha$, and IL-2. Extracellular pathogens, such as those involved in a bacterial infection, initiate a humoral immune response characterized by the activation of type $2 \mathrm{~T}$ lymphocytes (T2), which produce IL-4, IL-5, IL-6, and IL-10. T1 and T2 cell responses are mutually inhibitory 
(Zhao et al., 2012). The differences in cytokine modulation between our results and the cytokine expression profile in a report of reovirus-infected mice (Hazlett et al., 2003) are shown in Table 2. G-CSF is known for its specific effects on the proliferation, differentiation, and activation of hematopoietic cells. The expression of G-CSF was strikingly increased in conditions of S. hyicus infection (193.81-fold) compared to that in reovirus infection (2.1-fold). IL-6, which promotes T2 reaction, also showed increased expression in S. hyicus infection. IL-12 expression was decreased in $S$. hyicus infection and increased in reovirus infection. The differences between the two cytokine expression profiles also shows that IL-10, the factor from Treg cells, and IL-17, from Th17 cells, were down-regulated in S. hyicus infections and up-regulated in reovirus infections. This suggests a potential role for Treg and Th17 cells in suppressing Th1 activation and shifting the Th1/Th2 balance to Th2 activation.

Table 2. Changes in cytokine levels after infection with $S$. hyicus or reovirus (for all changes, $P<0.05$ ).

\begin{tabular}{lcc}
\hline Cytokines & & Fold Change \\
\cline { 2 - 3 } & S. hyicus & Reovirus \\
\hline G-CSF & 193.81 & 2.1 \\
GM-CSF & 0.85 & 3.5 \\
IL-2 & 0.94 & 1.3 \\
IL-3 & 0.81 & 1.2 \\
IL-4 & 0.97 & 1.1 \\
IL-5 & 0.86 & 3 \\
IL-6 & 6.2 & 2.2 \\
IL-9 & 0.69 & 2.6 \\
IL-10 & 0.88 & 7.3 \\
IL-12p70 & 0.77 & 3.3 \\
IL-13 & 0.81 & 1.7 \\
IL-17 & 0.96 & 4 \\
IFN-Y & 0.87 & 5.9 \\
MCP-1/CCL2 & 0.66 & 3 \\
MCP-5/CCL12 & 1.45 & 3.1 \\
RANTES/CCL5 & 0.84 & 1.1 \\
SCF & 1.34 & 2.6 \\
STNFR1 & 1.05 & 1.8 \\
TNF- $\alpha$ & 0.99 & 1.7 \\
VEGF & 0.5 & 1 \\
\hline
\end{tabular}

In terms of limitations, this study did not explore the underlying reason for the aforementioned differences any further. After a bacterial infection, defense mechanisms give rise to immune changes to protect the body by increasing IL-6, mediated by the cellular immune response, and G-CSF, mediated by the humoral immune response. However, the pathways that are activated that result in a sophisticated pattern of cytokine expression, and their genetic regulation remain unclear.

In conclusion, by applying cytokine antibody array analysis to $S$. hyicus-infected mice, the cytokines IL-6, KC, G-CSF, and IL-12p40p70 have been detected in the expression profile as potential biomarkers for $S$. hyicus infection. In the future, further analysis and tracking of these cytokines may aid in the prognosis of $S$. hyicus infection and the determination of an appropriate treatment for this condition.

\section{Conflicts of interest}

The authors declare no conflict of interest. 


\section{ACKNOWLEDGMENTS}

Research supported by the grants from the Science and Technology Department of Guangdong Province (\#2012A020200014 and \#2013B020202002), the Department of Agriculture of Guangdong Province (Yuenong \#2009-380), the Science and Information Technology Bureau of Guangzhou (\#12C14071615), and the Guangdong Academy of Agricultural Sciences (\#201313).

\section{REFERENCES}

Abajo A, Bitarte N, Zarate R, Boni V, et al. (2012). Identification of colorectal cancer metastasis markers by an angiogenesisrelated cytokine-antibody array. World J. Gastroenterol. 18: 637-645.

Ahrens P and Andresen LO (2004). Cloning and sequence analysis of genes encoding Staphylococcus hyicus exfoliative toxin types A, B, C, and D. J. Bacteriol. 186: 1833-1837.

Andresen L (2005). Production of exfoliative toxin by isolates of Staphylococcus hyicus from different countries. Vet. Rec. 157: 376-378.

Borrebaeck CA and Wingren C (2007). High-throughput proteomics using antibody microarrays: an update. Expert Rev. Mol. Diagn. 7: 673-686.

Bozic CR, Kolakowski LJ, Gerard NP, Garcia-Rodriguez C, et al. (1995). Expression and biologic characterization of the murine chemokine KC. J. Immunol. 154: 6048-6057.

Casanova C, Iselin L, von Steiger N, Droz S, et al. (2011). Staphylococcus hyicus bacteremia in a farmer. J. Clin. Microbiol. 49: 4377-4378.

Cheers C, Haigh AM, Kelso A, Metcalf D, et al. (1988). Production of colony-stimulating factors (CSFs) during infection: separate determinations of macrophage-, granulocyte-, granulocyte-macrophage-, and multi-CSFs. Infect. Immun. 56: 247-251.

Chenier S and Lallier L (2012). Acantholytic folliculitis and epidermitis associated with Staphylococcus hyicus in a line of white Leghorn laying chickens. Vet. Pathol. 49: 284-287.

Dalrymple SA, Alattery R, Aud DM, Krishna M, et al. (1996). Interleukin-6 is required for a protective immune response to systemic Escherichia coli infection. Infect. Immun. 64: 3231-3235.

de la Fuente AJ, Ferri EF, Tejerina F, Frandoloso R, et al. (2009). Cytokine expression in colostrum-deprived pigs immunized and challenged with Haemophilus parasuis. Res. Vet. Sci. 87: 47-52.

Demetri GD and Griffin JD (1991). Granulocyte colony-stimulating factor and its receptor. Blood 78: 2791-2808.

Fielding CA, McLoughlin RM, McLeod L, Colmont CS, et al. (2008). IL-6 regulates neutrophil trafficking during acute inflammation via STAT3. J. Immunol. 181: 2189-2195.

Fudaba Y, Nishifuji K, Andresen LO, Yamaguchi T, et al. (2005). Staphylococcus hyicus exfoliative toxins selectively digest porcine desmoglein 1. Microb. Pathog. 39: 171-176.

Futagawa-Saito K, Ba-Thein W, Higuchi T, Sakurai N, et al. (2007). Nationwide molecular surveillance of exfoliative toxigenic Staphylococcus hyicus on pig farms across Japan. Vet. Microbiol. 124: 370-374.

Hazlett LD, Huang X, McClellan SA and Barrett RP (2003). Further studies on the role of IL-12 in Pseudomonas aeruginosa corneal infection. Eye 17: 863-871.

Hill HR and Martins TB (2006). The flow cytometric analysis of cytokines using multi-analyte fluorescence microarray technology. Methods 38: 312-316.

Iyori K, Hisatsune J, Kawakami T, Shibata S, et al. (2010). Identification of a novel Staphylococcus pseudintermedius exfoliative toxin gene and its prevalence in isolates from canines with pyoderma and healthy dogs. FEMS Microbiol. Lett. 312: 169175.

Jiang W, Huang R, Duan C, Fu L, et al. (2013). Identification of five serum protein markers for detection of ovarian cancer by antibody arrays. PLOS ONE 8: e76795.

Jin KB, Choi HJ, Kim HT, Hwang EA, et al. (2008). Cytokine array after cyclosporine treatment in rats. Transplant Proc. 40: 2682-2684.

Kawakami M, Tsutsumi H, Kumakawa T, Abe H, et al. (1990). Levels of serum granulocyte colony-stimulating factor in patients with infections. Blood 76: 1962-1964.

Kawakami T, Ohashi S, Kawa Y, Takahama H, et al. (2004). Elevated serum granulocyte colony-stimulating factor levels in patients with active phase of sweet syndrome and patients with active behcet disease: implication in neutrophil apoptosis dysfunction. Arch. Dermatol. 140: 570-574. 
Kim HB, Kim CK, lijima K, Kobayashi T, et al. (2009). Protein microarray analysis in patients with asthma: elevation of the chemokine PARC/CCL18 in sputum. Chest 135: 295-302.

L'Ecuyer C and Jericho K (1966). Exudative epidermitis in pigs: etiological studies and pathology. Can. J. Comp. Med. Vet. Sci. 30: 94-101.

McDonald CL, Steinbach K, Kern F, Schweigreiter R, et al. (2011). Nogo receptor is involved in the adhesion of dendritic cells to myelin. J. Neuroinflammation 8: 113.

Nishifuji KM, Sugai M and Amagai M (2008). Staphylococcal exfoliative toxins: "molecular scissors" of bacteria that attack the cutaneous defense barrier in mammals. J. Dermatol. Sci. 49: 21-31.

Onogawa T, Saito-Taki T, Yamamoto H and Wada T (2013). IL6 trans-signaling promotes functional recovery of hypofunctional phagocytes through STAT3 activation during peritonitis. Inflamm. Res. 62: 797-810.

Osterlund A and Nordlund E (1997). Wound infection caused by Staphylococcus hyicus subspecies hyicus after a donkey bite. Scand. J. Infect. Dis. 29: 95.

Phillips WE Jr, King RE and Kloos WE (1980). Isolation of Staphylococcus hyicus subsp hyicus from a pig with septic polyarthritis. Am. J. Vet. Res. 41: 274-276.

Roberson JR, Fox LK, Hancock DD, Gay JM, et al. (1996). Prevalence of coagulase-positive staphylococci, other than Staphylococcus aureus, in bovine mastitis. Am. J. Vet. Res. 57: 54-58.

Rolle CE, Chen J, Pastar I, Cardenas TC, et al. (2013). Keratinocytes produce IL-6 in response to desmoglein 1 cleavage by Staphylococcus aureus exfoliative toxin A. Immunol. Res. 57: 258-267.

Tanabe T, Sato H, Sato H, Watanabe K, et al. (1996). Correlation between occurrence of exudative epidermitis and exfoliative toxin-producing ability of Staphylococcus hyicus. Vet. Microbiol. 48: 9-17.

Tosi MF (2005). Innate immune responses to infection. J. Allergy Clin. Immunol. 116: 241-249.

Une C, Andersson J and Orn A (2003). Role of IFN-alpha/beta and IL-12 in the activation of natural killer cells and interferongamma production during experimental infection with Trypanosoma cruzi. Clin. Exp. Immunol. 134: $195-201$.

van den Berg S, Laman JD, Boon L, ten Kate MT, et al. (2013). Distinctive cytokines as biomarkers predicting fatal outcome of severe Staphylococcus aureus bacteremia in mice. PLoS ONE 8: e59107.

Zhao G, Zhou S, Davie A and Su Q (2012). Effects of moderate and high intensity exercise on T1/T2 balance. Exerc. Immunol. Rev. 18: 98-114. 